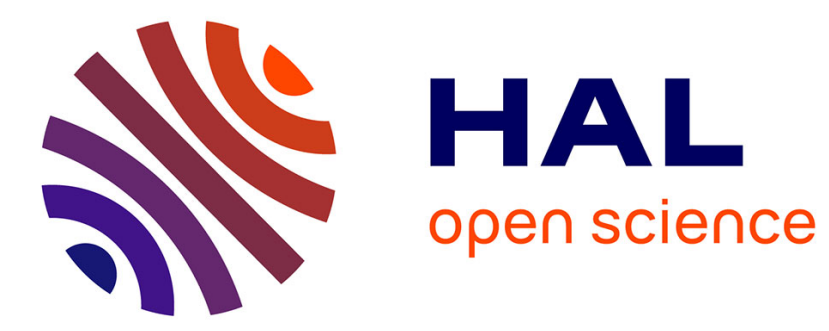

\title{
Probabilistic analysis of strip footings resting on spatially varying soils and subjected to vertical or inclined loads
}

\author{
Tamara Al-Bittar, Abdul-Hamid Soubra
}

\section{- To cite this version:}

Tamara Al-Bittar, Abdul-Hamid Soubra. Probabilistic analysis of strip footings resting on spatially varying soils and subjected to vertical or inclined loads. Journal of Geotechnical and Geoenvironmental Engineering, 2013, 140 (4), 10.1061/(ASCE)GT.1943-5606.0001046 . hal-01007214

\section{HAL Id: hal-01007214 https://hal.science/hal-01007214}

Submitted on 7 Jul 2018

HAL is a multi-disciplinary open access archive for the deposit and dissemination of scientific research documents, whether they are published or not. The documents may come from teaching and research institutions in France or abroad, or from public or private research centers.
L'archive ouverte pluridisciplinaire HAL, est destinée au dépôt et à la diffusion de documents scientifiques de niveau recherche, publiés ou non, émanant des établissements d'enseignement et de recherche français ou étrangers, des laboratoires publics ou privés. 


\title{
Probabilistic Analysis of Strip Footings Resting on Spatially Varying Soils and Subjected to Vertical or Inclined Loads
}

\author{
Tamara Al-Bittar ${ }^{1}$ and Abdul-Hamid Soubra ${ }^{2}$
}

\begin{abstract}
A probabilistic analysis of vertically and obliquely loaded strip footings resting on a spatially varying soil is presented. The system responses are the footing vertical and horizontal displacements. The deterministic computation of these system responses is based on numerical simulations using the software FLAC $3 D$. Both cases of isotropic and anisotropic random fields are considered for the soil elastic properties. The uncertainty propagation methodology employed makes use of a nonintrusive approach to build up analytical equations for the two system responses. Thus, a Monte Carlo simulation approach is applied directly on these analytical equations (not on the original deterministic model), which significantly reduces the computation time. In the case of the footing vertical load, a global sensitivity analysis has shown that the soil Young's modulus $E$ mostly contributes to the variability of the footing vertical displacement, the Poisson ratio being of negligible weight. The decrease in the autocorrelation distances of $E$ has led to a smaller variability of the footing displacement. On the other hand, the increase in the coefficient of variation of $E$ was found to increase both the probabilistic mean and the variability of the footing displacement. Finally, in the inclined loading case, the results of the probability of failure against exceedance of a vertical and/or a horizontal footing displacement are presented and discussed.
\end{abstract}

Keywords: Probabilistic analysis; Spatial variability; Shallow foundation; Footing displacement.

\section{Introduction}

The effect of the spatial variability of the soil elastic properties on the behavior of geotechnical structures was investigated by several authors (Paice et al. 1996; Fenton and Griffiths 2002; Nour et al. 2002; Jimenez and Sitar 2009; Elachachi et al. 2012, among others). For the problem of shallow foundations, only the footing vertical load case was considered in the analysis (see Paice et al. 1996; Fenton and Griffiths 2002, 2008; Jimenez and Sitar 2009). Also, only the case of an isotropic autocorrelation distance of the soil elastic properties is generally adopted although the soil is, in reality, anisotropic, because of the depositional and post-depositional processes. The present paper fills these gaps. It aims at investigating the probabilistic analysis of a strip footing resting on a spatially varying soil (where the soil Young's modulus $E$ and Poisson ratio $\nu$ were considered as anisotropic random fields with different values for their horizontal and vertical autocorrelation distances) and subjected to a vertical or an inclined load.

When dealing with a probabilistic analysis that involves spatially varying soil properties, Monte Carlo simulation (MCS) methodology is generally used to determine the probability density function (PDF) of the system response. This method is well known to be a computationally expensive approach. This is because it requires a great number of calls of the deterministic model (which

\footnotetext{
${ }^{1}$ Assistant Professor, Univ. of Balamand, Koura, North Lebanon, Lebanon (corresponding author). E-mail: tamara.albittar@balamand.edu.lb

${ }^{2}$ Professor, Univ. of Nantes, 44603 Saint-Nazaire, France. E-mail: abed .soubra@univ-nantes.fr
}

is generally based on a FEM or a finite-difference method). To overcome the inconvenience of the time cost, this paper presents a more efficient probabilistic approach called sparse polynomial chaos expansion (SPCE) (Blatman and Sudret 2010), which significantly reduces the number of calls of the deterministic model. Note that the sparse polynomial chaos expansion is an extension of the polynomial chaos expansion (PCE). A PCE or a SPCE methodology aims at replacing the deterministic model, which may be an analytical model or a finite-element/finite-difference model by a metamodel (i.e., a simple analytical equation). Within the framework of the PCE or the SPCE methodology, the PDF of the system response and/or the failure probability against a prescribed threshold can be easily obtained. This is because MCS is no longer applied on the original computationally expensive deterministic model, but on the metamodel. The other significant advantage of the present SPCE methodology is that it allows one to easily perform a global sensitivity analysis based on Sobol indexes. These indexes give the contribution of each random field to the variability of the system response.

In a first stage, the results of the vertical load case are presented: A global sensitivity analysis based on Sobol indexes was performed to detect the most influential soil elastic property $(E$ or $\nu)$ that has a significant weight in the variability of the system response. Then, a probabilistic parametric study was undertaken using only the random field, which has a significant weight in the variability of the system response. The aim of this study is to determine the effect of the different governing statistical characteristics (autocorrelation distances and coefficient of variation) of the most influential random field on the PDF of the footing vertical displacement. In a second stage, the numerical results of the inclined load case where the probabilities of failure against exceedance of prescribed vertical and/or horizontal footing displacements are presented and discussed.

The paper is organized as follows. The next section aims at presenting the probabilistic analysis. It is followed by the numerical results. The paper ends with a conclusion. 


\section{Probabilistic Analysis}

The aim of this section is the probabilistic analysis of vertically and obliquely loaded strip footings resting on a spatially varying soil. The system responses are the footing vertical and horizontal displacements. The deterministic computation of these system responses is based on numerical simulations using the software FLAC $3 D$. The soil Young's modulus $E$ and Poisson ratio $\nu$ were considered as two anisotropic random fields. They were characterized by a common square exponential autocorrelation function (see Popescu et al. 2005 ) and by two log-normal (LN) probability density functions. The log-normal distribution for $E$ and $\nu$ has been selected because of its mathematical convenience. The expansion optimal linear estimation (EOLE) method proposed by Li and Der Kiureghian (1993) was used to discretize the two random fields. The SPCE methodology was employed to approximate the system response by an analytical equation and to perform the probabilistic analysis. Finally, global sensitivity analysis based on Sobol indexes was performed to determine the weight of each random field in the variability of the system response.

\section{Discretization of a Nonisotropic Log-Normal Random Field}

Consider a two-dimensional nonisotropic log-normal random field $Z^{L N}$ described by a log-normal marginal cumulative distribution function $F_{G}$, and a square exponential autocorrelation function $\rho_{Z}^{L N}\left[(x, y),\left(x^{\prime}, y^{\prime}\right)\right]$ that gives the values of the correlation between two arbitrary points $(x, y)$ and $\left(x^{\prime}, y^{\prime}\right)$. Note that this function is given as follows:

$$
\rho_{Z}^{L N}\left[(x, y),\left(x^{\prime}, y^{\prime}\right)\right]=\exp \left[-\left(\frac{\left|x-x^{\prime}\right|}{a_{x}}\right)^{2}-\left(\frac{\left|y-y^{\prime}\right|}{a_{y}}\right)^{2}\right]
$$

where $a_{x}$ and $a_{y}=$ autocorrelation distances along $x$ and $y$, respectively. The EOLE method proposed by Li and Der Kiureghian (1993) to discretize a random field is used herein. In this method, one should first define a stochastic grid composed of $s$ grid points (or nodes) and determine the log-normal autocorrelation matrix $\sum^{L N}$, which gives the correlation between each grid point of the stochastic mesh and the other grid points of this mesh using Eq. (1). The lognormal autocorrelation matrix $\sum^{L N}$ should then be transformed into the Gaussian space using the Nataf transformation (see Nataf 1962). As a result, one obtains a Gaussian autocorrelation matrix $\sum^{G}$ that can be used to discretize the Gaussian random field $Z$ as follows:

$$
\tilde{Z}(x, y) \cong \mu_{\ln Z}+\sigma_{\ln Z} \sum_{j=1}^{N} \frac{\xi_{j}}{\sqrt{\lambda_{j}}} .\left(\phi_{j}\right)^{T} \Omega
$$

where $\mu_{\ln Z}$ and $\sigma_{\ln Z}=$ mean and SD values of the underlying normal distribution [i.e., $\ln (Z)] ;\left(\lambda_{j}, \phi_{j}\right)=$ eigenvalues and eigenvectors of the Gaussian autocorrelation matrix $\sum^{G} ; \Omega=$ correlation vector between the value of the field at an arbitrary point $(x, y)$ and its values at the different grid points; $\xi_{j}(j=1, \ldots, N)=$ vector of standard normal random variables; and $N=$ number of terms (expansion order) retained in the EOLE method. This number $N$ is obtained by sorting the eigenvalues $\lambda_{j}(j=1, \ldots, s)$ in a descending order, and by choosing the number $N$ of eigenmodes leading to a variance of the error that is smaller than a prescribed tolerance $\varepsilon(\varepsilon \approx 10 \%$ in this paper). Note that the variance of the error for EOLE is given by Li and Der Kiureghian (1993) as follows:

$$
\operatorname{Var}[Z(x, y)-\tilde{Z}(x, y)]=\sigma_{\ln Z}^{2}\left\{1-\sum_{j=1}^{N} \frac{1}{\lambda_{j}}\left[\left(\phi_{j}\right)^{T} \Omega\right]^{2}\right\}
$$

where $Z(x, y)$ and $\tilde{Z}(x, y)=$ exact and approximate values, respectively, of the random fields at a given point $(x, y)$; and $\left(\phi_{j}\right)^{T}=$ transpose of the eigenvector $\phi_{j}$. Note finally that $\left(\phi_{j}\right)^{T}$ and $\Omega$ in Eq. (3) are two vectors of dimensions $(1 \times s)$ and $(s \times 1)$, respectively.

Once the Gaussian random field is obtained, it should be transformed into the log-normal space by exponentiating the approximated Gaussian random field $\tilde{Z}(x, y)$ given by Eq. (2).

\section{Methodology Used for the Output Approximation}

In this section, one first presents the polynomial chaos expansion and then its extension, the sparse polynomial chaos expansion. The polynomial chaos expansion methodology allows one to approximate a complex deterministic model by an analytical equation (called hereafter the metamodel). Thus, the system response may be calculated (when performing the probabilistic analysis by MCS) using a simple analytical equation (not the original deterministic model). Within the PCE methodology, the system response $\Gamma$ of a deterministic model with $M$ random variables can be expressed by an analytical equation as follows:

$$
\Gamma_{\mathrm{PCE}}(\xi)=\sum_{\beta=0}^{\infty} a_{\beta} \Psi_{\beta}(\xi) \cong \sum_{\beta=0}^{P-1} a_{\beta} \Psi_{\beta}(\xi)
$$

where $P=$ number of terms retained in the truncation scheme; $\xi=\left\{\xi_{i}\right\}_{i=1, \ldots, M}=$ vector of $M$ independent standard random variables that represent the $M$ random variables; $a_{\beta}=$ unknown coefficients to be computed; and $\Psi_{\beta}=$ multivariate Hermite polynomials. These multivariate Hermite polynomials can be obtained from the product of one-dimensional (1D) Hermite polynomials as follows:

$$
\Psi_{\beta}=\prod_{i=1}^{M} H_{\alpha_{i}}\left(\xi_{i}\right)
$$

where $\alpha_{i}(i=1, \ldots, M)=$ sequence of $M$ nonnegative integers; and $H_{\alpha_{i}}()=.\alpha_{i}^{\text {th }} 1 \mathrm{D}$ Hermite polynomial. The expressions of the 1D Hermite polynomials are given in Appendix I. The coefficients $a_{\beta}$ of the PCE may be efficiently computed using a nonintrusive technique where the deterministic calculations are done using, for example, a finite-element/finite-difference software treated as a black box. The most used nonintrusive method is the regression approach (e.g., Isukapalli et al. 1998; Huang et al. 2009; Blatman and Sudret 2010; Li et al. 2011; Mollon et al. 2011, 2013; Houmadi et al. 2012; Ahmed and Soubra 2012; Al-Bittar and Soubra 2013). This method is used in the present work. The deterministic calculations used in this method are based (in this paper) on numerical simulations using FLAC 3D software.

In fact, for a PCE of order $p$, only the multivariate polynomials $\Psi_{\beta}$ of degree $\leq p$ should be retained. This leads to a number $P$ [see Eq. (4)] of the unknown PCE coefficients $=(M+p) ! / M ! p !$ !. It should be noted that the number of the PCE coefficients to be computed grows dramatically with the size $M$ of the input random vector and the PCE order $p$. When dealing with random fields as is the case in the present paper (and especially when considering small values of the autocorrelation distances), the discretization of the random fields by EOLE may lead to a significant number of random variables, which makes the determination of the PCE coefficients 
unfeasible because of the significant increase in the number of calls of the deterministic model. To address such a problem, the sparse polynomial chaos expansion developed by Blatman and Sudret (2010) is used herein. Indeed, Blatman and Sudret (2010) have shown that the number of significant terms in a PCE is relatively small because the multivariate polynomials $\Psi_{\beta}$ corresponding to high-order interaction (i.e., those resulting from the multiplication of the $H_{\alpha_{i}}$ with increasing $\alpha_{i}$ values) are associated with very small values for the coefficients $a_{\beta}$. Based on this observation, these authors have proposed a truncation strategy (called the hyperbolic truncation scheme) which suggests that the $q$-norm $\|\alpha\|_{q}$ should be less than or equal to the order $p$ of the PCE. The $q$-norm is given by

$$
\|\alpha\|_{q}=\left[\sum_{i=1}^{M}\left(\alpha_{i}\right)^{q}\right]^{1 / q}
$$

where $q=$ coefficient $(0<q<1)$. In this formula, $q$ can be chosen arbitrarily. Blatman and Sudret (2010) have shown that sufficient accuracy is obtained for $q \geq 0.5$.

The proposed SPCE methodology leads to a sparse polynomial chaos expansion that contains a small number of unknown coefficients, which can be calculated from a reduced number of calls of the deterministic model with respect to the classical PCE methodology. Note that the SPCE methodology as proposed by Blatman and Sudret (2010) is based on an iterative procedure to arrive to a minimal number for the SPCE coefficients. This procedure is used in this paper to build up an SPCE of the system response. For more details on this iterative procedure, the reader may refer to Blatman and Sudret (2010) [see also the flowchart presented in Al-Bittar (2012) and Al-Bittar and Soubra (2013)]. Once the coefficients $a_{\beta}$ have been computed, the PDF of the system response and the corresponding statistical moments (mean, SD, skewness, and kurtosis) can be calculated with no additional cost using the metamodel.

\section{Regression Approach}

Consider a set of $K$ realizations $\left\{\xi^{(1)}=\left(\xi_{1, \ldots,} \xi_{M}\right), \ldots, \xi^{(K)}\right.$ $\left.=\left(\xi_{1}, \ldots, \xi_{M}\right)\right\}$ of the standard normal random vector $\xi$. These realizations are called experimental design and can be obtained from Monte Carlo simulations. It is noted that $\Gamma=\left\{\Gamma\left(\xi^{(1)}\right), \ldots, \Gamma\left(\xi^{(K)}\right)\right\}$ is a vector containing the corresponding values of the response. The computation of the SPCE coefficients using the regression approach is performed using the following equation:

$$
\widehat{a}=\left(\eta^{T} \eta\right)^{-1} \eta^{T} \Gamma
$$

where the matrix $\eta$ is defined by

$$
\eta_{i \beta}=\Psi_{\beta}\left(\xi^{(i)}\right), \quad i=1, \ldots, K, \quad \beta=0, \ldots, J-1
$$

where $J=$ number of the retained SPCE coefficients. To ensure the numerical stability of the treated problem in Eq. (7), the size $K$ of the experimental design must be selected in such a way that the matrix $\left(\eta^{T} \eta\right)^{-1}$ is well-conditioned. This implies that the rank of this matrix should be larger than or equal to the number of unknown coefficients. This test was systematically performed while solving the linear system of equations of the regression approach. Note finally that the quality of the output approximation via an SPCE closely depends on the SPCE order $p$. To ensure a good fit between the metamodel and the true deterministic model (i.e., to obtain the optimal SPCE order), one successively increases the SPCE order until a prescribed accuracy was obtained. In this paper, the coefficient of determination $Q^{2}$ is used (see Blatman and Sudret 2010). This coefficient of determination is more efficient than the classical coefficient of determination $R^{2}$, because it allows one to check the metamodel's capability of correctly predicting the model response at any point that does not belong to the experimental design.

\section{Global Sensitivity Analysis}

Once the SPCE coefficients are determined, a global sensitivity analysis based on Sobol indexes can be easily performed. Note that the first-order Sobol index of a given random variable $\xi_{i}(i=1, \ldots, M)$ gives the contribution of this variable in the variability of the system response. The first-order Sobol index is given by Saltelli et al. (2000) and Sobol (2001) as follows:

$$
S\left(\xi_{i}\right)=\frac{\operatorname{var}\left[E\left(\Gamma \mid \xi_{i}\right)\right]}{\operatorname{var}(\Gamma)}
$$

where $\Gamma=$ system response; $E\left(\Gamma \mid \xi_{i}\right)=$ expectation of $\Gamma$ conditional on a fixed value of $\xi_{i}$; and var $=$ variance. In the present paper, the system response is represented by an SPCE. Thus, by replacing $\Gamma$ in Eq. (9) with the SPCE expression, one obtains the Sobol index formula as a function of the different terms of the SPCE (Sudret 2008). This formula is given by

$$
S\left(\xi_{i}\right)=\frac{\sum_{\beta \in I_{i}}\left(a_{\beta}\right)^{2} E\left[\left(\Psi_{\beta}\right)^{2}\right]}{\sum_{\beta=0}^{P-1}\left(a_{\beta}\right)^{2} E\left[\left(\Psi_{\beta}\right)^{2}\right]}
$$

where $a_{\beta}=$ obtained SPCE coefficients; $\Psi_{\beta}=$ multivariate Hermite polynomials; $E[]=$. expectation operator; and $E\left[\left(\Psi_{\beta}\right)^{2}\right]$ is given by Sudret (2008) as follows:

$$
E\left[\left(\Psi_{\beta}\right)^{2}\right]=\prod_{i=1}^{M} \alpha_{i} \text { ! }
$$

where $\alpha_{i}=$ same sequence of $M$ nonnegative integers $\left\{\alpha_{1}, \ldots, \alpha_{M}\right\}$ used in Eq. (5). Note, finally, that $I_{i}$, which appears in the numerator of Eq. (10), denotes the set of indexes $\beta$ for which the corresponding $\Psi_{\beta}$ terms are only functions of the random variable $\xi_{i}$ (i.e., they only contain the variable $\xi_{i}$ ). In the present paper where random fields are involved, the Sobol index of a random field is computed as the sum of the Sobol indexes of the different variables that represent this field.

To illustrate the construction of a PCE and the derivation of the equations providing Sobol indexes, an illustrative example of a PCE of order $p=3$ using only $M=2$ random variables $\left(\xi_{1}\right.$ and $\left.\xi_{2}\right)$ is presented in Appendix II.

\section{Numerical Results}

Once the coefficients $a_{\beta}$ have been computed using the regression approach, the PDF of the system response and/or the failure probability against a prescribed threshold can be calculated with no additional cost. This is performed by generating a large number of realizations of the standard normal random vector $\xi_{i}(i$ $=1, \ldots, M)$ and by computing the corresponding system responses using the metamodel. The following sections are devoted to the numerical results of both the vertically and obliquely loaded footings. 


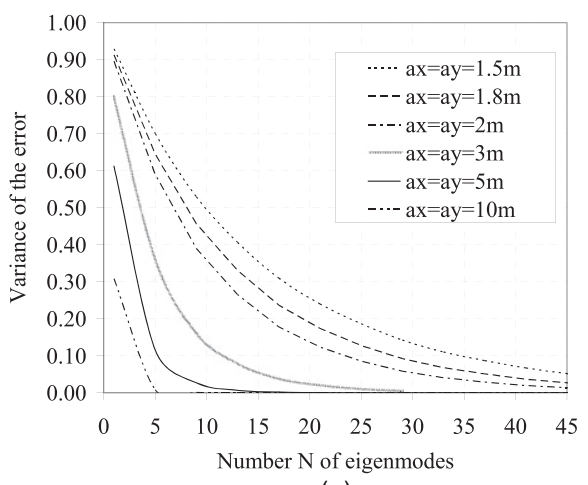

(a)

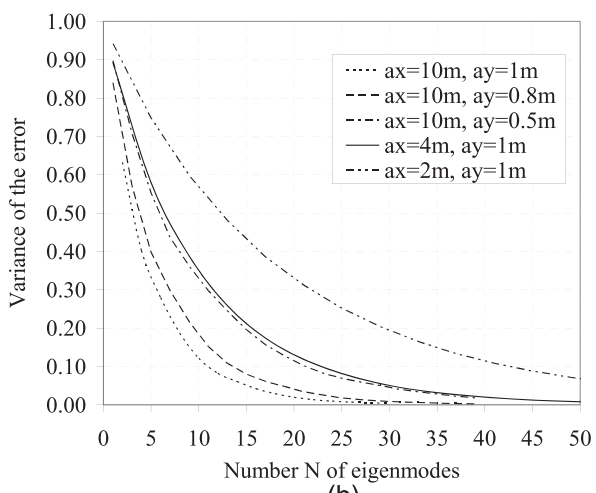

(b)

Fig. 1. Number $N$ of eigenmodes needed in the EOLE method: (a) isotropic case; (b) anisotropic case

\section{Case of a Vertically Loaded Footing}

The aim of this section is to present the probabilistic numerical results of a shallow strip footing resting on a spatially varying soil and subjected to a central vertical load $\left(P_{v}\right)$. Both the soil Young's modulus and Poisson ratio were firstly considered as random fields to determine the weight of each random field in the variability of the system response. Note that the same autocorrelation function (square exponential) was used for both random fields. The Young's modulus $E$ of the soil was assumed to be log-normally distributed. Its mean value and coefficient of variation (referred to in this paper as reference values) were taken as $\mu_{E}=60 \mathrm{MPa}, \mathrm{COV}_{E}=15 \%$. Similarly, the Poisson ratio $v$ was assumed to be log-normally distributed with a mean value and a coefficient of variation given as $\mu_{\nu}=0.3$, $\mathrm{COV}_{v}=5 \%$. As for the autocorrelation distances $a_{x}$ and $a_{y}$ of the soil random fields ( $E$ and $v$ ), both cases of isotropic (i.e., $a_{x}=a_{y}$ ) and anisotropic (i.e., $a_{x} \# a_{y}$ ) autocorrelation distances will be treated although the soil is rarely isotropic in reality. For the isotropic case, a range of 1.5-100 $\mathrm{m}$ was considered. For the anisotropic case, ElRamly et al. (2003) have shown that $a_{x}$ is within a range of $10-40 \mathrm{~m}$, while $a_{y}$ ranges from 1 to $3 \mathrm{~m}$. These values are in accordance with those given by Phoon and Kulhawy (1999). In our study, the reference values adopted for $a_{x}$ and $a_{y}$ were $a_{x}=10 \mathrm{~m}$ and $a_{y}=1 \mathrm{~m}$ while the wide ranges of 2-50 and 0.5-50 m were considered, respectively, for $a_{x}$ and $a_{y}$ when performing the parametric study. As may be seen from Fig. 1, for a given soil variability, the variance of the error decreases with the increase in the number $N$ of eigenmodes (i.e., random variables). For the different autocorrelation distances, the total number $N$ of random variables (or eigenmodes) that should be used to discretize the two random fields within the prescribed value of $10 \%$ for the variance of the error is presented in Table 1. As may be seen from this table, the smaller values of the autocorrelation distance $\left(a_{x}, a_{y}\right.$ or $\left.a_{x}=a_{y}\right)$ require a greater number of eigenmodes (random variables) to arrive to the prescribed value of the variance of the error.

The deterministic model was based on numerical simulations using the finite-difference code FLAC $3 D$. Even though a service load is considered, the soil behavior was modeled using a conventional elastic-perfectly plastic model based on a Mohr-Coulomb failure criterion to consider the plasticity that may occur at the footing corners. Note that the soil cohesion $c$, the soil angle of internal friction $\varphi$, and the soil dilation angle $\psi$ were assumed to be deterministic because the footing vertical displacement is not very sensitive to these variables. Their corresponding values were, respectively, $c=20 \mathrm{kPa}, \varphi=30^{\circ}$, and $\psi=20^{\circ}$. Concerning the footing, a weightless strip foundation of $2-\mathrm{m}$ width and $0.5-\mathrm{m}$ height
Table 1. Number of Random Variables Used to Discretize the Random Field $E$ or $v$ for both Cases of Isotropic and Anisotropic Autocorrelation Distances

\begin{tabular}{lc}
\hline & $\begin{array}{c}\text { Total number of random variables used } \\
\text { to discretize the Young's modulus or } \\
\text { the Poisson ratio random field }\end{array}$ \\
Values of $a_{x}$ and $a_{y}$ & \\
\hline Isotropic case & 35 \\
$a_{x}=a_{y}=1.5 \mathrm{~m}$ & 30 \\
$a_{x}=a_{y}=1.8 \mathrm{~m}$ & 25 \\
$a_{x}=a_{y}=2 \mathrm{~m}$ & 12 \\
$a_{x}=a_{y}=3 \mathrm{~m}$ & 10 \\
$a_{x}=a_{y}=5 \mathrm{~m}$ & 5 \\
$a_{x}=a_{y} \geq 10 \mathrm{~m}$ & \\
Anisotropic case & 12 \\
$a_{x}=10 \mathrm{~m}, a_{y} \geq 1 \mathrm{~m}$ & 15 \\
$a_{x}=10 \mathrm{~m}, a_{y}=0.8 \mathrm{~m}$ & 22 \\
$a_{x}=10 \mathrm{~m}, a_{y}=0.5 \mathrm{~m}$ & 24 \\
$a_{x}=4 \mathrm{~m}, a_{y}=1 \mathrm{~m}$ & 44 \\
$a_{x}=2 \mathrm{~m}, a_{y}=1 \mathrm{~m}$ & 12 \\
$a_{x} \geq 10 \mathrm{~m}, a_{y}=1 \mathrm{~m}$ &
\end{tabular}

was used. It was assumed to follow an elastic linear model ( $E$ $=25 \mathrm{GPa}, \nu=0.4)$. The connection between the footing and the soil mass was modeled by interface elements having the same mean values of the soil shear strength parameters to simulate a perfectly rough soil-footing interface. These parameters have been considered as deterministic in this study. Concerning the elastic properties of the interface, they also have been considered as deterministic and their values were $K_{s}=1 \mathrm{GPa}$ and $K_{n}=1 \mathrm{GPa}$, where $K_{s}$ and $K_{n}=$ shear and normal stiffness of the interface, respectively. Finally, note that the footing was subjected to a vertical applied pressure $q_{a}=500 \mathrm{kPa}$.

As shown in Fig. 2, the adopted soil domain considered in the analysis is $15 \mathrm{~m}$ wide $\times 6 \mathrm{~m}$ deep. It should be noted that the size of a given element in the deterministic mesh depends on the autocorrelation distances of the soil properties. Der Kiureghian and $\mathrm{Ke}$ (1988) have suggested that the length of the largest element of the deterministic mesh in a given direction (horizontal or vertical) should not exceed 0.5 times the autocorrelation distance in that direction. To respect this criterion for the different autocorrelation distances studied in this paper, two different deterministic meshes were considered in FLAC $3 D$ : the first one was devoted to the case of moderate to great values of the autocorrelation distances (i.e., when $a_{x} \geq 10 \mathrm{~m}$ and $a_{y} \geq 1 \mathrm{~m}$ ) [see Fig. 2(a)], and the second one 
for the small values of the autocorrelation distances [i.e., when $1.5 \mathrm{~m} \leq a_{x}<10 \mathrm{~m}$ or $0.5 \mathrm{~m} \leq a_{y}<1 \mathrm{~m}$; see Fig. 2(b)]. On the other hand, Li and Der Kiureghian (1993) have shown that the number of grid points in the stochastic mesh strongly depends on the autocorrelation distances. These authors have shown that a ratio of about $l_{R F} / a=1 / 5$ provides a sufficient accuracy in terms of the variance of the error where $l_{R F}$ is the typical element size in the stochastic grid and $a$ is the autocorrelation distance. This condition has been considered when constructing the stochastic mesh. For the boundary conditions of the deterministic mesh, the horizontal movement on the vertical boundaries of the grid was restrained, while the base of the grid was not allowed to move in both the horizontal and the vertical directions.

The procedure used herein to perform the probabilistic analysis is presented for the reference case study (i.e., when $a_{x}=10 \mathrm{~m}$ and $a_{y}=1 \mathrm{~m}$ ) considering both the Young's modulus $E$ and the Poisson ratio $v$ as random fields. A similar procedure was used when considering a single random field in the parametric study section. This procedure can be summarized as follows: one needs to discretize the two random fields $E$ and $v$ using the EOLE method. An arbitrary number of realizations $K=200$ (for each one of the two random fields $E$ and $v$ ) was performed using the MCS technique to construct the experimental design. The number $K$ is an initial (arbitrary) value because the iterative algorithm by Blatman and Sudret (2010) suggests to automatically add other simulations (an arbitrary number of $K^{\prime}=100$ realizations was taken in this paper) each time the regression problem is ill-posed. The use of a relatively large number of the added points is suggested to avoid several posttreatments because this becomes time-consuming. The algorithm stops if either the target accuracy $Q_{\text {TARGET }}^{2}$ is achieved or the SPCE order $p$ reached a maximal prescribed value fixed by the user. In this paper, a target accuracy $Q_{\text {TARGET }}^{2}=0.999$, a value of $q=0.7$ [in Eq. (6)], and a maximal SPCE order $=5$ were used. Note that for all the cases

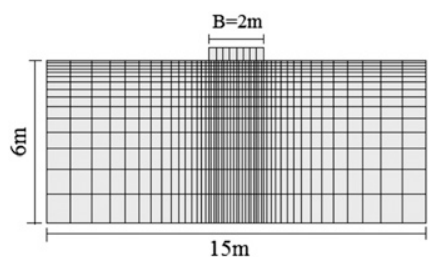

(a)

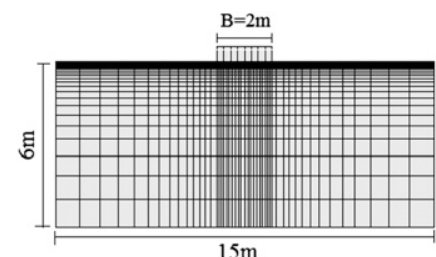

(b)
Fig. 2. Mesh used for the computation of the footing displacement: (a) for moderate to great values of the autocorrelation distances $\left(a_{x} \geq 10 \mathrm{~m}\right.$ and $a_{y} \geq 1 \mathrm{~m}$ ); (b) for small values of the autocorrelation distances $\left(a_{x}<10 \mathrm{~m}\right.$ or $\left.a_{y}<1 \mathrm{~m}\right)$ studied hereafter, the algorithm has stopped when the target accuracy $Q_{\text {TARGET }}^{2}$ was reached. The corresponding order of the SPCE was $=3$. For some illustrative autocorrelation distances used in the paper, the number of the classical PCE coefficients, the number of coefficients retained in the SPCE, and the number of calls of the deterministic model needed to construct this SPCE (i.e., metamodel) are presented in Table 2. From this table, one can see that the number of the retained coefficients increases when the autocorrelation distance decreases. Thus, the most significant time cost corresponds to the cases of largely heterogeneous soil masses. As may be seen from Table 2, a significant reduction in the number of coefficients (and consequently in the number of calls of the deterministic model) can be obtained using the SPCE. This greatly facilitates the solution of the problem of random fields.

Fig. 3 depicts (for the reference case study presented in Table 1 where $a_{x}=10 \mathrm{~m}$ and $a_{y}=1 \mathrm{~m}$ ) the values of Sobol indexes for the 24 random variables (12 random variables per random field). The first 12 random variables (i.e., $\xi_{i}$ for $i=1, \ldots, 12$ ) correspond to the Young's modulus random field and the last 12 random variables (i.e., $\xi_{i}$ for $\left.i=13, \ldots, 24\right)$ are those corresponding to the Poisson ratio random field. Fig. 3 shows that only three random variables $\left(\xi_{1}, \xi_{2}\right.$, and $\left.\xi_{4}\right)$ of the Young's modulus random field are the most influential (they involve $98.4 \%$ of the response variance). The Poisson ratio random field has a quasi-negligible weight in the variability of the system response $(0.14 \%$ of the system variance). For this reason, it can be considered as deterministic in the following section. This greatly facilitates the probabilistic analysis because it reduces by half the computation time.

It should be noted that although the three random variables $\left(\xi_{1}, \xi_{2}\right.$, and $\left.\xi_{4}\right)$ of the Young's modulus are the most influential, $\xi_{1}$ (which corresponds to the first eigenmode) is the most predominant one because it involves $94 \%$ of the response variance. Note that the first eigenmode is symmetrical with respect to the vertical axis of the footing. It has its maximal values along this vertical axis (figure not shown herein). The two other eigenmodes (corresponding to $\xi_{2}$ and $\xi_{4}$ ) provide small additional fluctuations to the first eigenmode and thus, they do not significantly contribute to the values of the Young's modulus random field at the different points of the soil mass. This observation can be explained by the fact that the system response (i.e., the vertical displacement) is a quantity that depends on the average distribution of the spatially varying Young's modulus over the entire domain and it is therefore quite insensitive to the smallscale fluctuations of the Young's modulus.

\section{Probabilistic Parametric Study}

The aim of this section is to study the effect of the different statistical governing parameters of the Young's modulus random field

Table 2. Number of Classical PCE Coefficients, Number of Coefficients Retained in the Sparse Polynomial Chaos Expansion, and Number of Calls of the Deterministic Model for both Cases of Isotropic and Anisotropic Autocorrelation Distances

\begin{tabular}{|c|c|c|c|}
\hline Values of $a_{x}$ and $a_{y}$ & $\begin{array}{l}\text { Number of the classical PCE } \\
\text { coefficients }\end{array}$ & $\begin{array}{l}\text { Number of coefficients retained in the } \\
\text { sparse polynomial chaos expansion }\end{array}$ & $\begin{array}{l}\text { Number of calls of the deterministic } \\
\text { model }\end{array}$ \\
\hline \multicolumn{4}{|l|}{ Isotropic } \\
\hline$a_{x}=a_{y}=1.5 \mathrm{~m}$ & 8,436 & 5,516 & 8,000 \\
\hline$a_{x}=a_{y}=100 \mathrm{~m}$ & 56 & 26 & 200 \\
\hline \multicolumn{4}{|l|}{ Anisotropic } \\
\hline$a_{x}=10 \mathrm{~m}, a_{y}=0.5 \mathrm{~m}$ & 2,300 & 1,340 & 2,000 \\
\hline$a_{x}=10 \mathrm{~m}, a_{y}=1 \mathrm{~m}$ & 455 & 186 & 300 \\
\hline$a_{x}=10 \mathrm{~m}, a_{y}=50 \mathrm{~m}$ & 455 & 26 & 200 \\
\hline$a_{x}=2 \mathrm{~m}, a_{y}=1 \mathrm{~m}$ & 16,215 & 8,500 & 10,000 \\
\hline$a_{x}=50 \mathrm{~m}, a_{y}=1 \mathrm{~m}$ & 455 & 147 & 200 \\
\hline
\end{tabular}




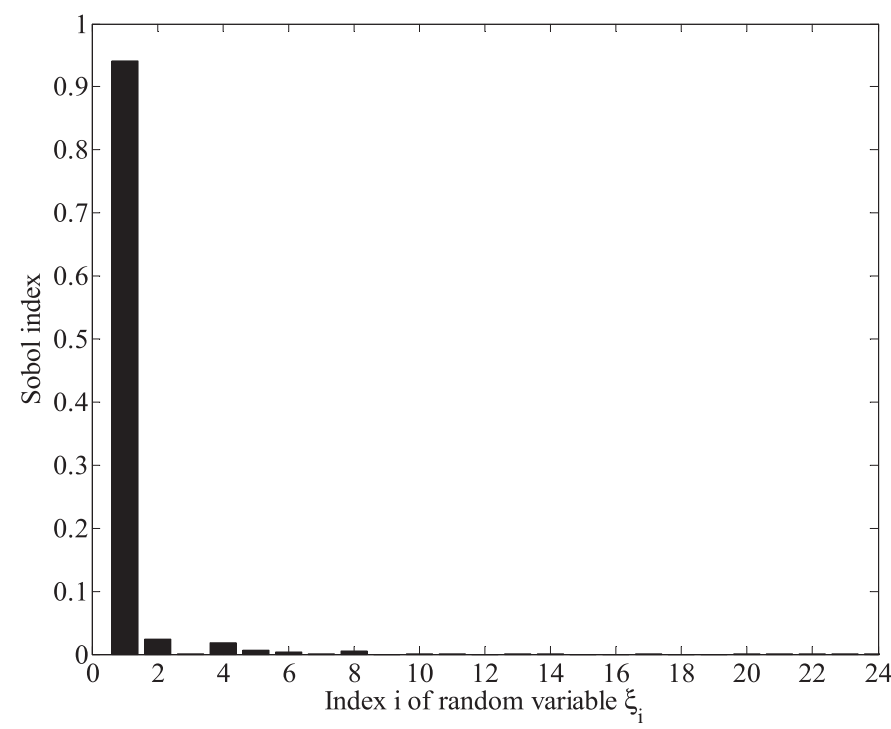

Fig. 3. Sobol indexes of the two random fields [the Young's modulus for $\xi_{i}(i=1, \ldots, 12)$ and the Poisson ratio for $\left.\xi_{i}(i=13, \ldots, 24)\right]$

(autocorrelation distances and coefficient of variation) on the PDF of the footing vertical displacement.

\section{Effect of the Autocorrelation Distance of the Young's Modulus: Isotropic Case}

Fig. 4 provides the PDFs of the footing vertical displacement for different values of the isotropic autocorrelation distance $a_{x}$ $=a_{y}\left(a_{x}=a_{y}=1.5,1.8,2,3,5,10,50\right.$, and $\left.100 \mathrm{~m}\right)$ and for the case of the random variable where the soil is assumed to be homogeneous with the same value of the coefficient of variation as that of the random field. Table 3 presents the four statistical moments for the cases presented in this figure. As expected, the PDF and the statistical moments corresponding to a great value of the autocorrelation distance $\left(a_{x}=a_{y}=100 \mathrm{~m}\right)$ are similar to those given by the case of random variable. This is because the case of a random variable can be considered as the limiting case of a random field with an infinite value of the autocorrelation distance.

Fig. 4 shows that the PDF is less spread out when the autocorrelation distance decreases. For the very large values of the isotropic autocorrelation distance $a_{x}=a_{y}=100 \mathrm{~m}$, the coefficient of variation of the footing vertical displacement tends to a constant maximal value (see Table 3 ), which is the value corresponding to the case of a random variable. In this case, the different values of the Young's modulus of a given realization are perfectly correlated. This means that for a given simulation, a single value of the Young's modulus affects the entire soil domain [see Fig. 5(a)]. This value is chosen according to the prescribed PDF of the Young's modulus and thus it may vary in the range of values imposed by this PDF. This leads to a large variability of the footing vertical displacement. The large value of the variability is because one obtains a large variety of low, intermediate, and high values of the soil Young's modulus from simulation to another one. The decrease in the autocorrelation distances from infinity to a finite value (moderate or small where $a_{x}=a_{y} \leq 10 \mathrm{~m}$ ) limits the correlation (in a given simulation) to a finite zone, which leads to several zones with different values of the Young's modulus over the entire soil domain. This means that in a single simulation, one obtains a set of weak and strong zones for which the position may change from simulation to another one [see Fig. 5(b)]. The case of moderate-to-small values of $a_{x}=a_{y}$ leads to

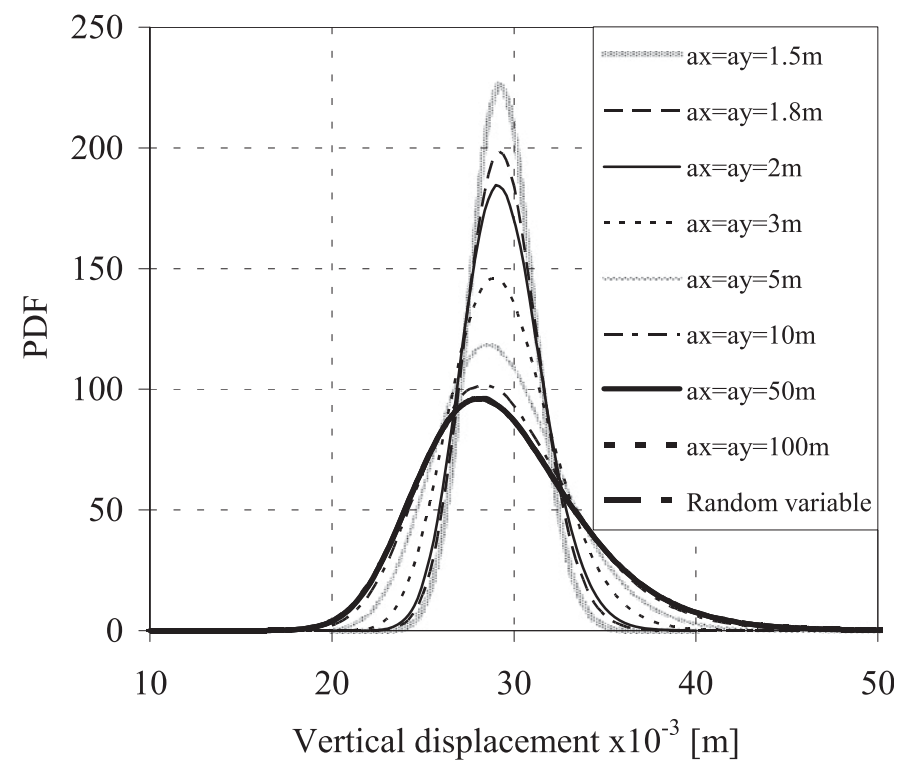

Fig. 4. Influence of the isotropic autocorrelation distance $a_{x}=a_{y}$ on the PDF of the footing vertical displacement

Table 3. Effect of Isotropic Autocorrelation Distance $a_{x}=a_{y}$ on Statistical Moments $\left(\mu, \sigma, \delta_{u}\right.$, and $\left.\kappa_{u}\right)$ of Footing Vertical Displacement

\begin{tabular}{lccccc}
\hline Values of $a_{x}$ and $a_{y}$ & $\begin{array}{c}\mu \times 10^{-3} \\
(\mathrm{~m})\end{array}$ & $\begin{array}{c}\sigma \times 10^{-3} \\
(\mathrm{~m})\end{array}$ & $\mathrm{COV} \%$ & $\delta_{u}$ & $\kappa_{u}$ \\
\hline$a_{x}=a_{y}=1.5 \mathrm{~m}$ & 29.4 & 1.8 & 6.12 & 0.0929 & 0.0121 \\
$a_{x}=a_{y}=1.8 \mathrm{~m}$ & 29.4 & 2.0 & 6.80 & 0.1877 & 0.0515 \\
$a_{x}=a_{y}=2 \mathrm{~m}$ & 29.4 & 2.2 & 7.48 & 0.2301 & 0.0737 \\
$a_{x}=a_{y}=3 \mathrm{~m}$ & 29.4 & 2.8 & 9.52 & 0.3268 & 0.1493 \\
$a_{x}=a_{y}=5 \mathrm{~m}$ & 29.5 & 3.5 & 11.86 & 0.3909 & 0.2760 \\
$a_{x}=a_{y}=10 \mathrm{~m}$ & 29.5 & 4.1 & 13.90 & 0.4344 & 0.3279 \\
$a_{x}=a_{y}=50 \mathrm{~m}$ & 29.5 & 4.4 & 14.92 & 0.4682 & 0.4054 \\
$a_{x}=a_{y}=500 \mathrm{~m}$ & 29.5 & 4.4 & 14.92 & 0.4684 & 0.4103 \\
Random variable & 29.5 & 4.4 & 14.92 & 0.4680 & 0.4100 \\
\hline
\end{tabular}

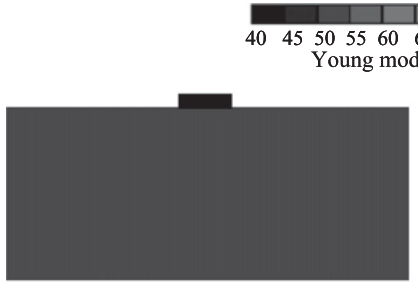

(a) $a_{x}=100 \mathrm{~m}$ and $a_{v}=100 \mathrm{~m}$

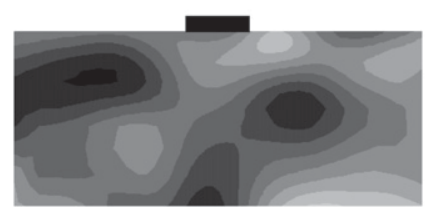

(c) $a_{x}=1.5 \mathrm{~m}$ and $a_{v}=1.5 \mathrm{~m}$

Fig. 5. Typical realizations of the Young's modulus random field for three values of the isotropic autocorrelation distance: (a) $a_{x}=100 \mathrm{~m}$ and $a_{y}=100 \mathrm{~m}$; (b) $a_{x}=3 \mathrm{~m}$ and $a_{y}=3 \mathrm{~m}$; (c) $a_{x}=1.5 \mathrm{~m}$ and $a_{y}=1.5 \mathrm{~m}$ 
a decrease in the variability of the footing vertical displacement because the cases of very high or very small footing vertical displacement are now absent, and because the presence of the soil heterogeneity (zones of weak and strong soil) will produce a somewhat close global behavior of the footing movement because of the averaging phenomenon over the zone influenced by the footing [see Fig. 5(c)]. The decrease in the variability of the footing vertical displacement becomes the most significant for the case of a very small value of the autocorrelation distance because the rapid change in the values of the Young's modulus from one element to another one of the soil mesh leads to quasi-similar footing displacements for all the realizations. The soil can be considered as an homogeneous medium in this case.

Table 3 shows that the probabilistic mean value of the footing vertical displacement does not exhibit a minimum and it remains constant regardless of the value of the autocorrelation distance $a_{x}=a_{y}$ (this mean value is found to be slightly greater than the deterministic value of $28.8 \mathrm{~mm}$, which makes it more critical). The nonpresence of a minimum is contrary to the probabilistic results of the ultimate bearing capacity problem (see Fenton and Griffiths 2003) where a minimum exists for a given value of the autocorrelation distance. This phenomenon can be explained by the fact that, under the service loads, the applied footing pressure $q_{a}=500 \mathrm{kPa}$ is not sufficiently high to induce or initiate a failure mechanism, which may pass through the weakest zones for a given value of the autocorrelation distance. Thus, in the present analysis, there is no particular value of the autocorrelation distance for which the soil exhibits some weakness with respect to the other values of the autocorrelation distance. Table 3 also gives the impact of the autocorrelation distance $a_{x}=a_{y}$ on both the skewness and the kurtosis of the PDF. For small values of $a_{x}=a_{y}$, the skewness and kurtosis of the response are close to zero, which means that the PDF of the response is not far from a Gaussian one in these cases. Note, however, that these moments increase when $a_{x}=a_{y}$ increases, which means that for great values of $a_{x}=a_{y}$, the shape of the PDF of the output becomes far from a Gaussian one.

\section{Effect of the Autocorrelation Distances of the Young's Modulus: Anisotropic Case}

Fig. 6 presents the PDFs of the footing vertical displacement for different values of $a_{y}\left(a_{y}=0.5,0.8,1,2,5,8\right.$, and $\left.50 \mathrm{~m}\right)$ when $a_{x}=10 \mathrm{~m}$, and for the case of a $1 \mathrm{D}$ horizontal random field when $a_{x}=10 \mathrm{~m}$. Table 4 presents the corresponding four statistical moments. Similarly, Fig. 7 presents the PDFs of the footing vertical displacement for different values of $a_{x}\left(a_{x}=2,4,10,20,30\right.$, and $50 \mathrm{~m}$ ) when $a_{y}=1 \mathrm{~m}$, and for the case of a $1 \mathrm{D}$ vertical random field when $a_{y}=1 \mathrm{~m}$. Table 5 presents the corresponding four statistical moments.

For the very large values of the autocorrelation distance $a_{x}$ or $a_{y}$, the coefficient of variation of the footing vertical displacement tends to a constant maximal value, which corresponds to the value obtained in the case of a 1D (vertical or horizontal) random field as may be seen from Tables 4 and 5. In this case, the Young's modulus is perfectly correlated in a single direction (horizontal or vertical); in contrast, the other direction is allowed to exhibit variations in the value of the Young's modulus according to the value of the autocorrelation distance fixed for that direction. This leads to a horizontal or a vertical multilayer, as may be seen from Figs. 8( $\mathrm{a}$ and $\mathrm{b}$ ). Although the case of a vertical multilayer is not a practical case, it is presented herein:

1. In the aim to check the validity of the obtained solutions by showing that for great values of $a_{y}$, one obtains the case of a 1D random field; and

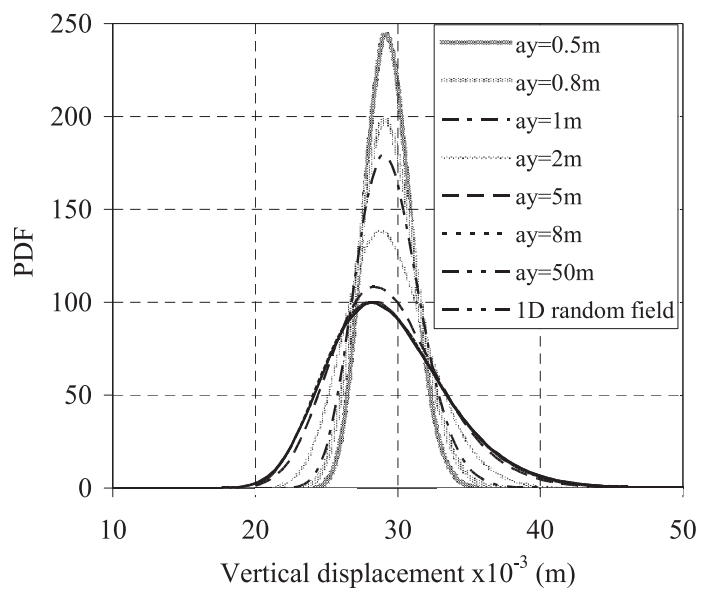

Fig. 6. Influence of the vertical autocorrelation distance $a_{y}$ on the PDF of the footing vertical displacement in the case where $a_{x}=10 \mathrm{~m}$

Table 4. Effect of Vertical Autocorrelation Distance $a_{y}$ on Statistical Moments $\left(\mu, \sigma, \delta_{u}\right.$, and $\left.\kappa_{u}\right)$ of Footing Vertical Displacement When $a_{x}=10 \mathrm{~m}$

\begin{tabular}{lccccc}
\hline \multicolumn{7}{c}{$\begin{array}{c}\mu \times 10^{-3} \\
(\mathrm{~m})\end{array}$} & $\begin{array}{c}\sigma \times 10^{-3} \\
(\mathrm{~m})\end{array}$ & $\mathrm{COV} \%$ & $\delta_{u}$ & $\kappa_{u}$ \\
\hline 0.5 & 29.3 & 1.6 & 5.46 & 0.1712 & 0.0329 \\
0.8 & 29.4 & 2.0 & 6.80 & 0.2077 & 0.0581 \\
1 & 29.4 & 2.2 & 7.48 & 0.2443 & 0.0841 \\
2 & 29.4 & 3.0 & 10.20 & 0.3312 & 0.2111 \\
5 & 29.5 & 3.8 & 12.88 & 0.4098 & 0.3094 \\
8 & 29.4 & 4.1 & 13.95 & 0.4178 & 0.3101 \\
50 & 29.5 & 4.2 & 14.24 & 0.4480 & 0.3374 \\
1D random field & 29.5 & 4.2 & 14.24 & 0.4480 & 0.3370 \\
\hline
\end{tabular}

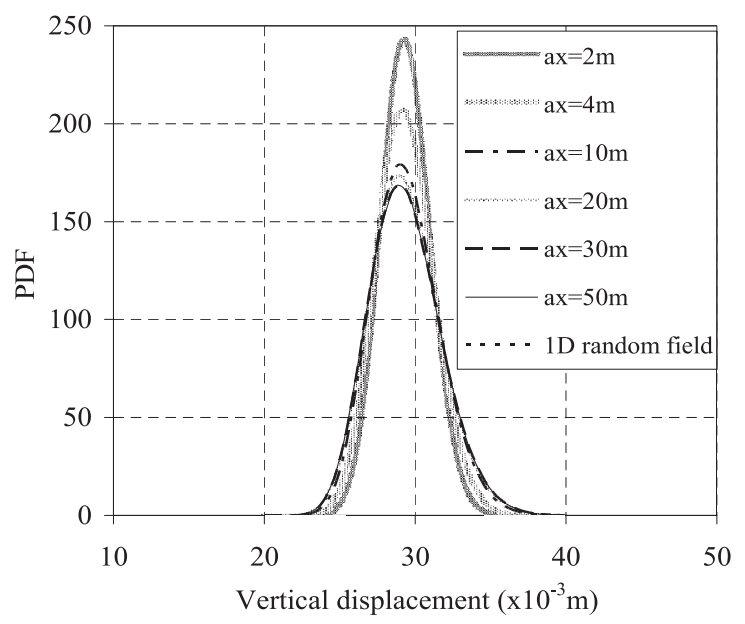

Fig. 7. Influence of the horizontal autocorrelation distance $a_{x}$ on the PDF of the footing vertical displacement in the case where $a_{y}=1 \mathrm{~m}$

2. To facilitate the interpretation of the obtained results, as will be shown in the next section.

The values of 14.24 and 8.16 (see Tables 4 and 5) concerning the variability of the $1 \mathrm{D}$ random fields are smaller than the value of 14.92 (see Table 3), corresponding to the case of random variable. This is because, contrarily to the random variable case where the Young's 
Table 5. Effect of Horizontal Autocorrelation Distance $a_{x}$ on Statistical Moments $\left(\mu, \sigma, \delta_{u}\right.$, and $\left.\kappa_{u}\right)$ of Footing Vertical Displacement When $a_{y}=1 \mathrm{~m}$

\begin{tabular}{lccccc}
\hline & $\begin{array}{c}\mu \times 10^{-3} \\
(\mathrm{~m})\end{array}$ & $\begin{array}{c}\sigma \times 10^{-3} \\
(\mathrm{~m})\end{array}$ & $\mathrm{COV} \%$ & $\delta_{u}$ & $\kappa_{u}$ \\
\hline 2 & 29.4 & 1.6 & 5.44 & 0.0914 & 0.0167 \\
4 & 29.4 & 1.9 & 6.46 & 0.1611 & 0.0475 \\
10 & 29.4 & 2.2 & 7.48 & 0.2443 & 0.0841 \\
20 & 29.4 & 2.4 & 8.16 & 0.2632 & 0.0952 \\
30 & 29.4 & 2.4 & 8.16 & 0.2667 & 0.0981 \\
50 & 29.4 & 2.4 & 8.16 & 0.2538 & 0.0999 \\
1D random field & 29.4 & 2.4 & 8.16 & 0.2600 & 0.1230 \\
\hline
\end{tabular}

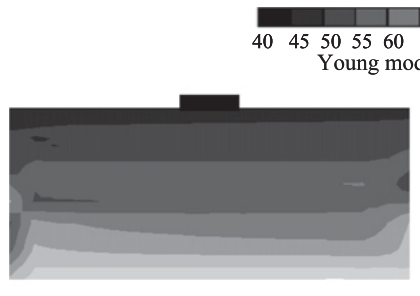

(a) $a_{x}=100 \mathrm{~m}$ and $a_{y}=1 \mathrm{~m}$

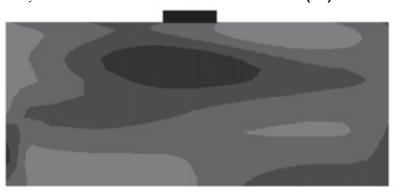

(c) $a_{x}=5 \mathrm{~m}$ and $a_{y}=1 \mathrm{~m}$

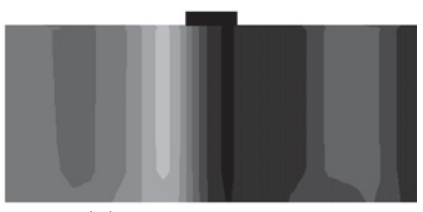

(b) $a_{x}=1 \mathrm{~m}$ and $a_{y}=100 \mathrm{~m}$
Fig. 8. Typical realizations of the Young's modulus random field for three values of the anisotropic autocorrelation distance: (a) $a_{x}=100 \mathrm{~m}$ and $a_{y}=1 \mathrm{~m}$; (b) $a_{x}=1 \mathrm{~m}$ and $a_{y}=100 \mathrm{~m}$; (c) $a_{x}=5 \mathrm{~m}$ and $a_{y}=1 \mathrm{~m}$

modulus of each simulation is chosen from the PDF where small, high, and intermediate values of the Young's modulus lead to a large variability, in the present case of 1D random field the horizontal or vertical strong layers prevent the large footing movement and lead to quasi-similar smaller footing displacement and thus to a smaller variability of this displacement. Finally, the decrease in the autocorrelation distance from infinity (i.e., from the case of a 1D random field) to a finite value [see Fig. 8(c)] recreates variation in the value of the Young's modulus, which reduces once again the value of the variability of this displacement. On the other hand, Tables 4 and 5 show that the probabilistic mean value of the footing vertical displacement is constant regardless of the value of the autocorrelation distance $\left(a_{x}\right.$ or $\left.a_{y}\right)$. The same explanation done for the isotropic case remains valid herein. Tables 4 and 5 also show the impact of the increase in $a_{y}$ or $a_{x}$ on both the skewness and the kurtosis of the PDF. As in the case of the isotropic autocorrelation distance, the PDF of the response is not far from a Gaussian one for small values of $a_{y}$ or $a_{x}$.

\section{Effect of the Coefficient of Variation of the Young's Modulus}

Fig. 9 presents the PDFs of the footing vertical displacement for four different values of the coefficient of variation of the Young's modulus random field. Note that for these four configurations, $a_{x}$ $=10 \mathrm{~m}$ and $a_{y}=1 \mathrm{~m}$. Table 6 presents (for the four configurations) the four statistical moments of the footing vertical displacement. As expected, Fig. 9 shows that the variability of the footing vertical displacement increases when the coefficient of variation of the

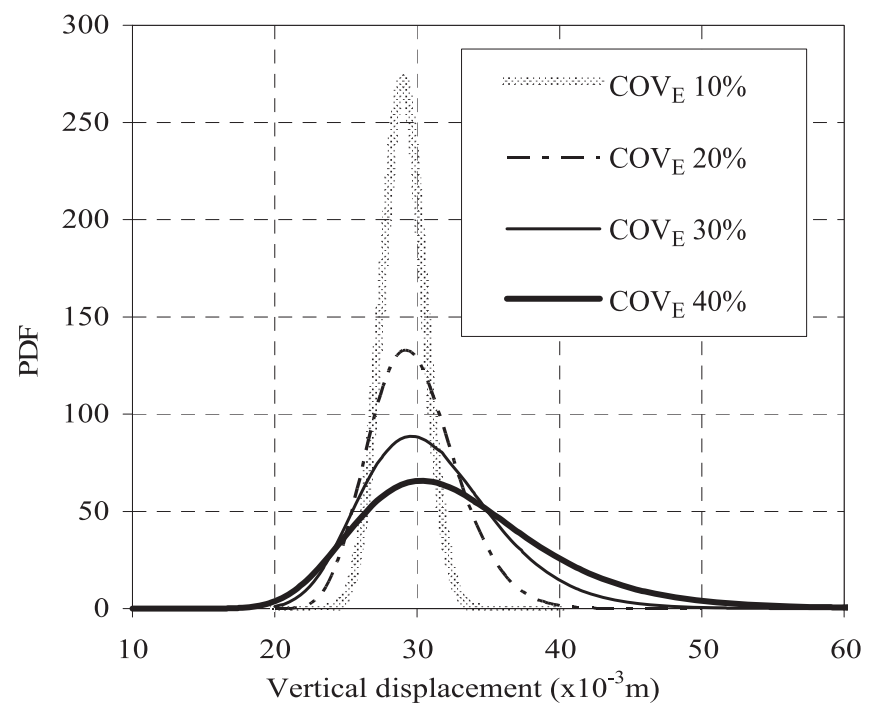

Fig. 9. Influence of the coefficient of variation $\mathrm{COV}_{E}$ on the PDF of the footing vertical displacement in the case where $a_{x}=10 \mathrm{~m}$ and $a_{y}=1 \mathrm{~m}$

Table 6. Effect of Coefficient of Variation $\left(\mathrm{COV}_{E}\right)$ of the Random Field $E$ on the Statistical Moments $\left(\mu, \sigma, \delta_{u}\right.$, and $\left.\kappa_{u}\right)$ of Footing Vertical Displacement when $a_{x}=10 \mathrm{~m}, a_{y}=1 \mathrm{~m}$

\begin{tabular}{lccrcl}
\hline $\operatorname{COV}_{E}(\%)$ & $\mu \times 10^{-3}(\mathrm{~m})$ & $\sigma \times 10^{-3}(\mathrm{~m})$ & $\mathrm{COV} \%$ & $\delta_{u}$ & \multicolumn{1}{c}{$\kappa_{u}$} \\
\hline 10 & 29.0 & 1.5 & 5.17 & 0 & 0 \\
20 & 29.8 & 3.0 & 10.07 & 0.3222 & 0.1456 \\
30 & 31.1 & 4.7 & 15.11 & 0.4883 & 0.4070 \\
40 & 32.9 & 6.6 & 20.06 & 0.6534 & 0.7791 \\
\hline
\end{tabular}

Young's modulus random field increases (see also Table 6). The mean value of the footing vertical displacement was found to significantly increase when the coefficient of variation of the Young's modulus random field increases. This is of particular interest, because the probabilistic mean value obtained for the reference case where $\mathrm{COV}_{E}=15 \%$ becomes unconservative and no longer valid when the variability of the input random field significantly increases. Table 6 also gives that for the smallest value of $\operatorname{COV}_{E}$ (i.e., $\mathrm{COV}_{E}=10 \%$ ), the skewness and kurtosis of the response are equal to zero, which means that the PDF of the response is Gaussian in this case. Note, however, that when $\mathrm{COV}_{E}$ increases, the shape of the PDF of the output becomes far from a Gaussian one.

\section{Case of an Obliquely Loaded Footing}

The aim of this section is to present the probabilistic numerical results obtained from the analysis of a shallow strip footing resting on a spatially varying soil and subjected to an inclined load. Only the reference case (i.e., $a_{x}=10 \mathrm{~m}, a_{y}=1 \mathrm{~m}$, and $\mathrm{COV}_{E}=15 \%$ ) was considered in the analysis. Note that the same deterministic values that were used in the vertical loading case were also used herein. The footing was subjected to an inclined load $P_{a}=400 \mathrm{kN} / \mathrm{m}$. The value of the inclination angle considered in this section was equal to $10^{\circ}$ with respect to the vertical axis of the footing.

Figs. 10 $(a$ and $b)$ present the PDFs of the footing vertical and horizontal displacements obtained by making use of the SPCE expressions (i.e., the metamodels) of the footing vertical and horizontal displacements. The metamodels of the footing vertical and horizontal displacements have also been used in this section to 


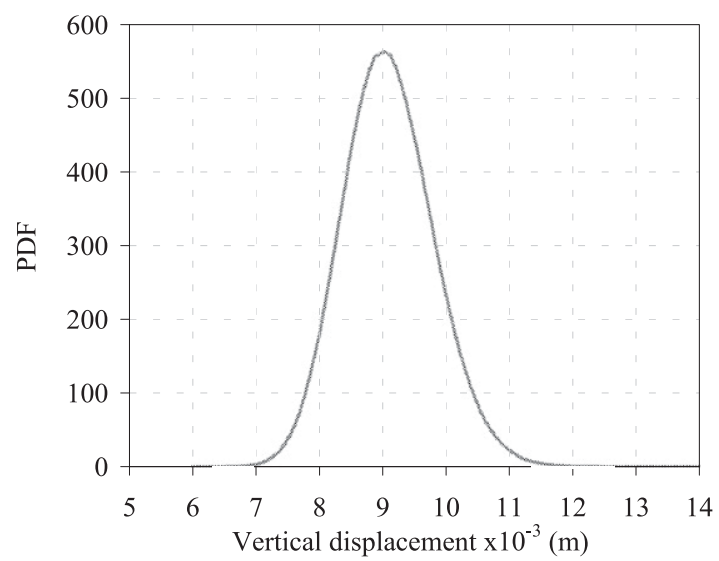

(a)

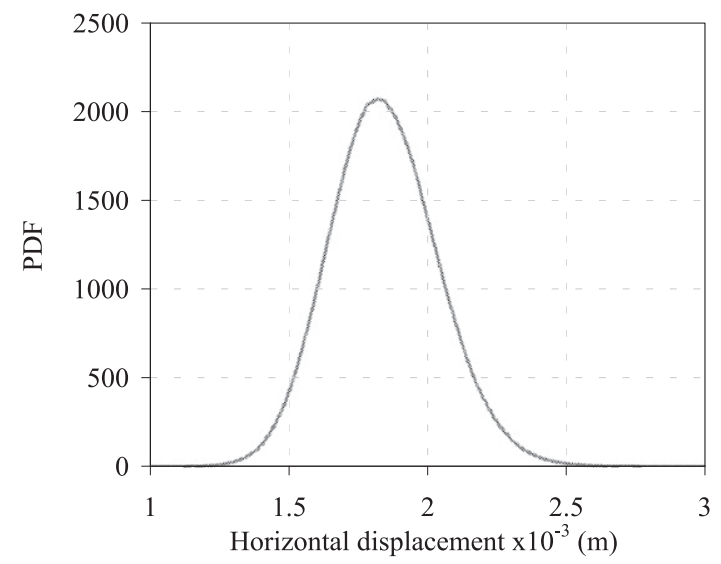

(b)

Fig. 10. PDF of (a) footing vertical displacement; (b) footing horizontal displacement in the case of an inclined load

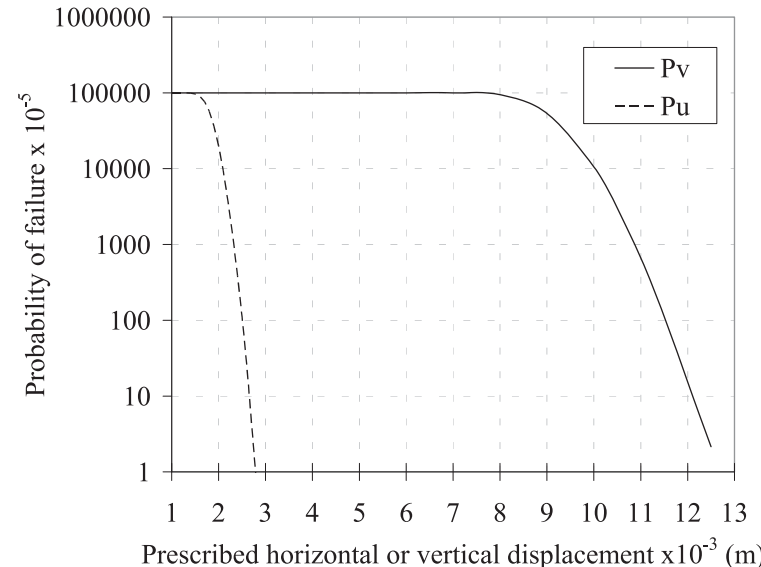

Fig. 11. Probability of exceeding a maximum vertical displacement (denoted $P_{v}$ ) or a maximum horizontal displacement (denoted $P_{u}$ )

compute the probability of exceeding of admissible horizontal and/ or vertical displacements. In the following, the probability of exceeding the admissible horizontal displacement $u_{\max }$ and the admissible vertical displacement $v_{\max }$ will be denoted, respectively, by $P_{u}$ and $P_{v}$. The probability of exceeding the admissible horizontal or vertical displacement will be referred to here as the system failure probability, and will denoted $P_{\text {sys }}$.

Fig. 11 shows the variation of $P_{u}$ and $P_{v}$ with the corresponding maximal displacement (i.e., $u_{\max }$ or $v_{\max }$ ). In this figure, each curve represents a single mode of failure (i.e., exceeding of a prescribed horizontal displacement or exceeding of a prescribed vertical displacement). Fig. 12 depicts the variation of $P_{\text {sys }}$ with $v_{\max }$ for four different values of $u_{\max }$. For the very small values of $v_{\max }$, the system failure probability $P_{\text {sys }}$ was found equal to $P_{v}$. For the small values of $v_{\max }$, the resulting number of simulations that respect the criterion $v>v_{\max }$ is relatively large, the number of simulations that correspond to $u>u_{\max }$ being negligible. In these cases, the failure mode corresponding to $P_{v}$ is predominant. As the value of $v_{\max }$ increases, the number of simulations corresponding to $v>v_{\max }$ was found to diminish. Furthermore, the value of $P_{\text {sys }}$ was found to diminish until reaching a value of $\left(P_{\text {sys }}\right)_{c r}$, beyond which the system failure probability remains constant. This may be explained by the predominance of the mode of failure corresponding to $P_{u}$ for these

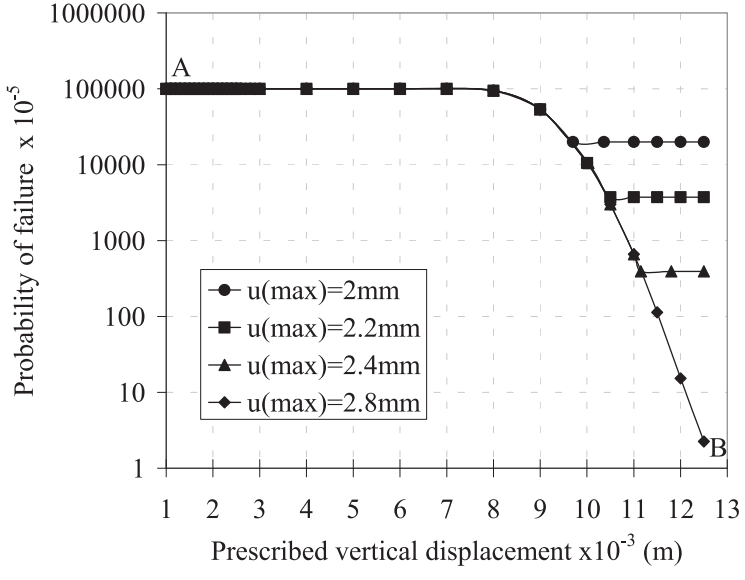

Fig. 12. Probability of exceeding a maximum vertical or horizontal displacement (denoted $P_{\text {sys }}$ )

cases. The reason why $P_{\text {sys }}$ becomes constant is the constant value considered for the maximal horizontal displacement $u_{\max }$. Note that the value of $v_{\max }$ for which one obtains $\left(P_{\text {sys }}\right)_{c r}$ depends on the value of $u_{\max }$. This value of $v_{\max }$ increases with the increase in $u_{\max }$ (see, for instance, the three values of $u_{\max }=2,2.2$, and $2.4 \mathrm{~mm}$ and the corresponding values of $v_{\max }=9.7,10.5$, and $11.1 \mathrm{~mm}$ ). For the large value of $u_{\max }\left(u_{\max }=2.8 \mathrm{~mm}\right)$, the system failure probability is equal to $P_{v}$, which means that a unique mode of failure corresponding to $P_{v}$ is predominant in this case.

\section{Conclusions}

A probabilistic analysis of vertically and obliquely loaded strip footings, which considers the spatial variability of the soil elastic properties, was herein presented. The soil Young's modulus and Poisson ratio were modeled by random fields. The deterministic model was based on numerical simulations using FLAC3D. An efficient uncertainty propagation methodology that makes use of a nonintrusive approach to build up analytical equations for the footing displacements was employed. In the case of a vertically loaded footing, a global sensitivity analysis was performed to detect the most influential soil elastic property that has a significant weight in the variability of the footing vertical displacement. It was shown 
Table 7. Basis $\Psi_{\beta}(\beta=0, \ldots, 9)$ of PCE and Values of $E\left[\left(\Psi_{\beta}\right)^{2}\right]$ for a PCE with $M=2$ and $p=3$

\begin{tabular}{lclr}
\hline$\beta$ & Order of the term $\Psi_{\beta}$ & \multicolumn{1}{c}{$\Psi_{\beta}=\prod_{i=1}^{M} H_{\alpha_{i}}\left(\xi_{i}\right)$} & $E\left[\left(\Psi_{\beta}\right)^{2}\right]=\prod_{i=1}^{M} \alpha_{i} !$ \\
\hline 0 & $p=0$ & $\Psi_{0}=H_{0}\left(\xi_{1}\right) \times H_{0}\left(\xi_{2}\right)=1$ & $\alpha_{1} ! \times \alpha_{2} !=0 ! \times 0 !=1$ \\
1 & 1 & $\Psi_{1}=H_{1}\left(\xi_{1}\right) \times H_{0}\left(\xi_{2}\right)=\xi_{1}$ & $\alpha_{1} ! \times \alpha_{2} !=1 ! \times 0 !=1$ \\
2 & & $\Psi_{2}=H_{0}\left(\xi_{1}\right) \times H_{1}\left(\xi_{2}\right)=\xi_{2}$ & $\alpha_{1} ! \times \alpha_{2} !=0 ! \times 1 !=1$ \\
3 & 2 & $\Psi_{3}=H_{1}\left(\xi_{1}\right) \times H_{1}\left(\xi_{2}\right)=\xi_{1} \xi_{2}$ & $\alpha_{1} ! \times \alpha_{2} !=1 ! \times 1 !=1$ \\
4 & & $\Psi_{4}=H_{2}\left(\xi_{1}\right) \times H_{0}\left(\xi_{2}\right)=\xi_{1}^{2}-1$ & $\alpha_{1} ! \times \alpha_{2} !=2 ! \times 0 !=2$ \\
5 & & $\Psi_{5}=H_{0}\left(\xi_{1}\right) \times H_{2}\left(\xi_{2}\right)=\xi_{2}^{2}-1$ & $\alpha_{1} ! \times \alpha_{2} !=0 ! \times 2 !=2$ \\
6 & 3 & $\Psi_{6}=H_{2}\left(\xi_{1}\right) \times H_{1}\left(\xi_{2}\right)=\left(\xi_{1}^{2}-1\right) \xi_{2}$ & $\alpha_{1} ! \times \alpha_{2} !=2 ! \times 1 !=2$ \\
7 & & $\Psi_{7}=H_{1}\left(\xi_{1}\right) \times H_{2}\left(\xi_{2}\right)=\xi_{1}\left(\xi_{2}^{2}-1\right)$ & $\alpha_{1} ! \times \alpha_{2} !=1 ! \times 2 !=2$ \\
8 & & $\Psi_{8}=H_{3}\left(\xi_{1}\right) \times H_{0}\left(\xi_{2}\right)=\xi_{1}^{3}-3 \xi_{1}$ & $\alpha_{1} ! \times \alpha_{2} !=3 ! \times 0 !=3$ \\
9 & & $\Psi_{9}=H_{0}\left(\xi_{1}\right) \times H_{3}\left(\xi_{2}\right)=\xi_{2}^{3}-3 \xi_{2}$ & $\alpha_{1} ! \times \alpha_{2} !=0 ! \times 3 !=6$ \\
\hline
\end{tabular}

that the soil Young's modulus $E$ mostly contributes to the variability of the footing vertical displacement, the Poisson ratio being of negligible weight. Thus, only the Young's modulus was considered as a random field in the parametric study.

The probabilistic parametric study has shown that the variability and the probabilistic mean value of the footing vertical displacement increase with the increase in the coefficient of variation of the soil Young's modulus. With a decrease in the autocorrelation distance ( $a_{x}$ or $a_{y}$ or $a_{x}=a_{y}$ ) of the Young's modulus, a less spread-out PDF of the footing vertical displacement was obtained; however, the probabilistic mean value of the vertical displacement was found to remain constant regardless of the value of the autocorrelation distance in both the isotropic and the anisotropic cases. Small values of the autocorrelation distances lead to small values of skewness and kurtosis of the system response. Thus, a PDF of the system response that is not far from a Gaussian one was obtained in these cases. On the other hand, it was shown in the inclined loading case that the probability of failure against exceedance of a vertical and/or a horizontal footing displacement may be obtained with a reduced computation cost using the obtained SPCE expressions of the system responses. Finally, it should be mentioned that the present probabilistic analysis has the limitation of not considering the variability of the soil properties in the out-of-plane direction. This additional variability significantly increases the number of random variables and thus the number of calls of the deterministic model. Consequently, a more advanced probabilistic approach is needed in this case.

\section{Appendix I. 1D Hermite Polynomials}

The 1D Hermite polynomials are given by

$$
\begin{aligned}
& H_{0}(\xi)=1, \\
& H_{1}(\xi)=\xi, \\
& H_{2}(\xi)=\xi^{2}-1, \\
& H_{3}(\xi)=\xi^{3}-3 \xi \\
& H_{4}(\xi)=\xi^{4}-6 \xi^{2}+3, \\
& H_{5}(\xi)=\xi^{5}-10 \xi^{3}+15 \xi, \\
& H_{6}(\xi)=\xi^{6}-14 \xi^{4}+45 \xi^{2}-15, \\
& \vdots \\
& H_{n}(\xi)=\xi H_{n-2}(\xi)-H_{n-1}(\xi)
\end{aligned}
$$

\section{Appendix II. Illustrative Example}

To illustrate the PCE theory in a simple manner, a PCE of order $p=3$ using only $M=2$ random variables $\left(\xi_{1}\right.$ and $\xi_{2}$ ) will be considered in this illustrative example. As may be easily seen from Table 7, the PCE basis contains $P=10$ terms whose expressions $\Psi_{\beta}(\beta$ $=0, \ldots, 9)$ are computed using Eq. (5).

By using Table 7, one can write the PCE as a function of the input random variables $\left(\xi_{1}\right.$ and $\left.\xi_{2}\right)$ as follows:

$$
\begin{aligned}
\Gamma_{\mathrm{PCE}}(\xi)= & a_{0} \Psi_{0}+a_{1} \Psi_{1}+\cdots+a_{9} \Psi_{9} \\
= & a_{0}+a_{1} \xi_{1}+a_{2} \xi_{2}+a_{3} \xi_{1} \xi_{2}+\mathrm{a}_{4}\left(\xi_{1}^{2}-1\right)+a_{5}\left(\xi_{2}^{2}-1\right) \\
& +a_{6}\left(\xi_{1}^{2}-1\right) \xi_{2}+a_{7} \xi_{1}\left(\xi_{2}^{2}-1\right)+a_{8}\left(\xi_{1}^{3}-3 \xi_{1}\right) \\
& +a_{9}\left(\xi_{2}^{3}-3 \xi_{2}\right)
\end{aligned}
$$

where the unknown coefficients can be computed using Eq. (7). Once the PCE coefficients are computed, the first-order Sobol indexes for the two random variables $\left(\xi_{1}\right.$ and $\left.\xi_{2}\right)$ can be easily obtained using Eq. (10). The only additional step is to compute $E\left[\left(\Psi_{\beta}\right)^{2}\right]$ corresponding to these two random variables. Table 7 provides the values of $E\left[\left(\Psi_{\beta}\right)^{2}\right]$ computed using Eq. (11) for the different $\Psi_{\beta}$ terms. The expressions of the first-order Sobol indexes of the two random variables $\xi_{1}$ and $\xi_{2}$ can thus be written as follows:

$$
\begin{aligned}
& S\left(\xi_{1}\right)=\frac{a_{1}^{2}+2 a_{4}^{2}+6 a_{8}^{2}}{a_{1}^{2}+a_{2}^{2}+a_{3}^{2}+2 a_{4}^{2}+2 a_{5}^{2}+2 a_{6}^{2}+2 a_{7}^{2}+6 a_{8}^{2}+6 a_{9}^{2}}, \\
& S\left(\xi_{2}\right)=\frac{a_{2}^{2}+2 a_{5}^{2}+6 a_{9}^{2}}{a_{1}^{2}+a_{2}^{2}+a_{3}^{2}+2 a_{4}^{2}+2 a_{5}^{2}+2 a_{6}^{2}+2 a_{7}^{2}+6 a_{8}^{2}+6 a_{9}^{2}}
\end{aligned}
$$

where $I_{1}=(1,4,8)$; and $I_{2}=(2,5,9)$.

\section{References}

Ahmed, A., and Soubra, A. H. (2012). "Extension of subset simulation approach for uncertainty propagation and global sensitivity analysis." Georisk, 6(3), 162-176.

Al-Bittar, T. (2012). "Probabilistic analysis of shallow foundations resting on spatially varying soils." Ph.D. thesis, Univ. of Nantes, Nantes, France.

Al-Bittar, T., and Soubra, A. H. (2013). "Bearing capacity of strip footing on spatially random soils using sparse polynomial chaos expansion." Int. J. Numer. Anal. Meth. Geomech., 37(13), 2039-2060.

Blatman, G., and Sudret, B. (2010). "An adaptive algorithm to build up sparse polynomial chaos expansions for stochastic finite element analysis." Probab. Eng. Mech., 25(2), 183-197.

Der Kiureghian, A., and Ke, J. B. (1988). "The stochastic finite element method in structural reliability." Probab. Eng. Mech., 3(2), 83-91.

Elachachi, S. M., Breysse, D., and Denis, A. (2012). "The effects of soil spatial variability on the reliability of rigid buried pipes." Comput. Geotech., 43, 61-71. 
El-Ramly, H., Morgenstern, N. R., and Cruden, D. M. (2003). "Probabilistic stability analysis of a tailing dyke on presheared clayshale." Can. Geotech. J., 40(1), 192-208.

Fenton, G. A., and Griffiths, D. V. (2002). "Probabilistic foundation settlement on spatially random soil." J. Geotech. Geoenviron. Eng., 10.1061/(ASCE)1090-0241(2002)128:5(381), 381-391.

Fenton, G. A., and Griffiths, D. V. (2003). "Bearing capacity prediction of spatially random $c-\varphi$ soils." Can. Geotech. J., 40(1), 54-65.

Fenton, G. A., and Griffiths, D. V. (2008). Risk assessment in geotechnical engineering, Wiley, New York.

FLAC3D [Computer software]. Minneapolis, ITASCA Consulting Group.

Houmadi, Y., Ahmed, A., and Soubra, A. H. (2012). "Probabilistic analysis of a one-dimensional soil consolidation problem." Georisk, 6(1), 36-49.

Huang, S. P., Liang, B., and Phoon, K. K. (2009). "Geotechnical probabilistic analysis by collocation-based stochastic response surface method: An Excel add-in implementation." Georisk, 3(2), 75-86.

Isukapalli, S. S., Roy, A., and Georgopoulos, P. G. (1998). "Stochastic response surface methods (SRSMs) for uncertainty propagation: Application to environmental and biological systems." Risk Anal., 18(3), 351-363.

Jimenez, R., and Sitar, N. (2009). "The importance of distributions types on finite element analyses of foundation settlement." Comput. Geotech., 36(3), 474-483.

Li, C. C., and Der Kiureghian, A. (1993). "Optimal discretization of random fields.” J. Eng. Mech., 10.1061/(ASCE)0733-9399(1993)119:6(1136), 1136-1154.

Li, D., Chen, Y., Lu, W., and Zhou, C. (2011). "Stochastic response surface method for reliability analysis of rock slopes involving correlated nonnormal variables." Comput. Geotech., 38(1), 58-68.
Mollon, G., Dias, D., and Soubra, A. H. (2011). "Probabilistic analysis of pressurized tunnels against face stability using collocation-based stochastic response surface method." J. Geotech. Geoenviron. Eng., 10.1061/(ASCE)GT.1943-5606.0000443, 385-397.

Mollon, G., Dias, D., and Soubra, A. H. (2013). "Range of the safe retaining pressures of a pressurized tunnel face by a probabilistic approach." J. Geotech. Geoenviron. Eng., 10.1061/(ASCE)GT.1943-5606.0000911, 1954-1967.

Nataf, A. (1962). "Détermination des distributions de probabilités dont les marges sont données." Comptes Rendus de l'Académie des Sciences, 225(1), 42-43 (in French).

Nour, A., Slimani, A., and Laouami, N. (2002). "Foundation settlement via finite element analysis." Comput. Geotech., 29(8), 641-672.

Paice, G. M., Griffiths, D. V., and Fenton, G. A. (1996). "Finite element modeling of settlements on spatially random soil." J. Geotech. Engrg., 10.1061/(ASCE)0733-9410(1996)122:9(777), 777-779.

Phoon, K. K., and Kulhawy, F. H. (1999). "Characterization of geotechnical variability." Can. Geotech. J., 36(4), 612-624.

Popescu, R., Deodatis, G., and Nobahar, A. (2005). "Effect of random heterogeneity of soil properties on bearing capacity." Probab. Eng. Mech., 20(4), 324-341.

Saltelli, A., Chan, K., and Scott, E. M. (2000). Sensitivity analysis, Wiley, New York.

Sobol, I. M. (2001). "Global sensitivity indices for nonlinear mathematical models and their Monte Carlo estimates." Mech. Comput. Sim., 55(1-3), 271-280.

Sudret, B. (2008). "Global sensitivity analysis using polynomial chaos expansion.” Reliab. Eng. Syst. Saf., 93(7), 964-979. 